\title{
Tissue-engineered anterior segment eye cultures display an intraocular pressure homeostatic response
}

Susannah Waxman, Chao Wang, Yalong Dang, Ralitsa Loewen, Nils A. Loewen*

*Corresponding Author: Nils A. Loewen, MD, PhD

ORCID: 0000-0001-7167-1213

Department of Ophthalmology, University of Würzburg

Josef-Schneider-Straße 11

97080 Würzburg, Germany

Email: loewen.nils@gmail.com

Phone: +49-931-20120351, +49-931-20120245

Grant information: National Eye Institute K08EY022737 (NAL); Initiative to Cure Glaucoma of the Eye and Ear Foundation of Pittsburgh (NAL); Wiegand Fellowship of the Eye and Ear Foundation of Pittsburgh (YD); P30-EY08098 (NAL); Department grant by Research to Prevent Blindness (NAL); an unrestricted fellowship grant from the Xiangya Hospital of Central South University (SC).

Keywords: glaucoma, tissue engineering, trabecular meshwork, outflow facility 
Glaucoma is a blinding disease largely caused by increased resistance to drainage of fluid from the eye's anterior chamber, resulting in elevated intraocular pressure (IOP). A major site of fluid outflow regulation and pathology is the trabecular meshwork (TM), an extracellular matrix (ECM)-rich tissue at the entrance of the eye's drainage system. We aimed to characterize the structural and functional properties of a newly developed tissue-engineered (TE) anterior segment eye culture model. We hypothesized that repopulation of decellularized TM ECM with non-native TM cells could restore intraocular pressure (IOP) homeostatic ability. Decellularized porcine anterior segment scaffolds demonstrated complete removal of cells, significant reduction of DNA content, and well-preserved ECM ultrastructure. Seeded cells localized to the TM region ( $p<0.001$ ) and progressively infiltrated meshwork ECM. Cells reached a distribution comparable to control TM after four days of perfusion culture. After perfusion rate increase challenge, TE cultures maintained healthy IOPs through regulation of outflow resistance (reseeded $=16.53 \pm 0.89$, decellularized $=35.23 \pm 2.20 \mathrm{mmHg}, \mathrm{p}<0.0001$ ). In conclusion, we describe a readily available, storable, and biocompatible scaffold for anterior segment perfusion culture of non-native cells. TE organs demonstrated physiological similarities to native tissues and may reduce the need for scarce donor globes in outflow research. 


\section{Introduction}

Glaucoma, a leading cause of irreversible blindness, is a progressive optic neuropathy affecting over 70 million people worldwide ${ }^{1}$. Clear fluid in the eye's anterior chamber, called aqueous humor, is produced within the confines of the globe at a rate of 2 to $3 \mu \mathrm{L} / \mathrm{min}^{2,3}$. In healthy eyes, aqueous humor outflow is in equilibrium with production. Elevated IOP in glaucoma (above $21 \mathrm{mmHg}$ in primary open angle glaucoma ${ }^{4,5}$ ) is primarily caused by increased resistance to outflow. Increased IOP is an experimentally demonstrated cause of glaucoma ${ }^{6}$ and remains the only factor shown to prevent or reduce glaucomatous retinal ganglion cell death and consequent vision loss ${ }^{7,8}$.

As a primary site of outflow resistance and outflow tract pathogenesis, the trabecular meshwork (TM) is a target of great therapeutic interest. TM tissue is a dynamic, multilayer, filter-like structure that responds to environmental signals like mechanical strain and shear forces. TM extracellular matrix (ECM) turnover and remodeling are heavily involved in healthy IOP maintenance ${ }^{9-13}$.

TM cultures in vitro studies miss many defining features of glaucoma, such as pressure and flow. TM in vitro perfusion models have been constructed for this reason ${ }^{14,15}$. However, these models cannot replicate the complex layers of ECM fibers and beams throughout the thickness of native meshwork. Cell physiology in vitro is often significantly different from the same cells placed in a 3D substrate ${ }^{16-19}$, limiting translational capacity. Ex vivo perfused anterior segment models have been used to examine and manipulate TM function under more physiological, pressurized 3D conditions and from a range of species ${ }^{20-26}$. Unfortunately, human donor anterior segments are a limiting resource in outflow research. On the other hand, TM cells from donor globes, TM-removal surgeries, and TM cell lines, can be more often readily available 27,28 , lending themselves to more scalable studies.

As done in other tissues for both research ${ }^{29-34}$ and medical applications ${ }^{35-38}$, removal of cells from ECM can provide a bioartificial scaffold for recellularization with cells of choice. Sodium dodecyl sulfate (SDS) has been highly effective for removal of cellular material in various ECM-rich tissues while hallmark TM ECM components like collagen, elastin, and laminin, are mostly well conserved $29,39-41$. 
The purpose of this study was to test the IOP homeostatic ability of a newly developed tissue-engineered (TE) anterior segment organ culture model. We hypothesized that repopulation of decellularized TM with non-native TM cells could restore IOP homeostasis. The porcine anterior segment scaffold described here is accessible, storable, biocompatible, and free of porcine cells. When reseeded with porcine TM cells, TE cultures demonstrate localization of cells to the TM, full-depth cell infiltration of TM ECM, and an IOP homeostatic response similar to standard ex vivo organ cultures and in vivo eyes.

\section{Results}

\section{Scaffold production}

\section{Freeze/thaw + surfactant perfusion created decellularized AS scaffolds}

Freeze/thaw cycling ( $F /$ th) lysed all native cells, as shown previously ${ }^{42}$. $F /$ th and perfusion of standard perfusion media resulted in a reduction of nuclei in the TM, accumulation of basophilic, DAPI-positive aggregate in TM ECM, and retention of cell debris in the sclera and cornea (Fig. 1A). Freeze/thaw + surfactant immersion/agitation (IA) reduced cells in the tissue periphery. IA often resulted in loose-appearing meshwork and physical disruption of tissue, creating debris in samples (Fig. 1B). No difference in cellularity was seen between IA samples after 1,2 , and 5 days of SDS incubation. F/th + surfactant perfusion (P) created anterior segment scaffolds free of nuclei and debris with a well-preserved ECM structure.

DAPI signal in each treatment-group corresponded with hematoxylin staining. In F/th and IA, DAPI fluorescence intensity was not significantly different from control (Fig. 1D, $p=$ 0.387 and 0.575 , respectively.) DAPI signal in P was significantly lower than control ( $p=0.010$, 99.3\% reduction.) P scaffolds were used for subsequent experiments. Decellularized scaffolds could be refrozen at $-80^{\circ} \mathrm{C}$ and kept for as long as two months prior to reinstallation into the perfusion system.

\section{IOP physiological throughout decellularization}

IOP remained within a physiological range throughout the perfusion-decellularization process, indicating that the ECM was not subjected to any abnormal physical stresses (Fig. 2, n 
= 15.) After 24 hours of washing, scaffold IOP remained stable (within a $0.6 \mathrm{mmHg}$ range) through the duration of perfusion. Washed scaffolds appeared grossly normal and had transparent corneas. 24 scaffolds were produced. Due to hardware error, IOP recordings for 8 eyes were unavailable. One eye was contaminated during perfusion, removed from the perfusion system, and was not included in the IOP analysis.

\section{Scaffold reseeding}

\section{Seeded cells localized to the TM region}

The fluorescent signal of eGFP-expressing CrFK cells was visualized at all time-points. Fluorescence at 24, 48, and 144 hours post-seeding was localized in the TM region. At 24 hours, some fluorescence was seen in the corneal region; this was not observed at subsequent timepoints. Fluorescence was occasionally seen where the ciliary body had been attached. The TM was significantly brighter than other regions by an average of $90.1 \pm 7.5 \%(p<0.001$, Fig. 3$)$.

\section{Cell infiltration of the TM increased with time in perfusion culture}

The depth of cells in the TM was measured and compared to control at each time-point (supplementary material 1). The average depth of cells in the TM increased over time until its peak at four days (Fig. 4, Table 1). CrFK cell depth at 24 and 48 hours was significantly lower than control ( $p<0.05$ ) while the depth of TM cells at 96 and CrFK cells at 144 hours was not significantly different $(p=0.19,0.06)$. At 24 hours post-seeding, some cells could be seen on the basement membrane, consistent with fluorescence in Fig. 3. Cells migrated in the direction of outflow over time. Additionally, cellularity of the TE TM tended to increase with time (Table 1), indicating cell division ${ }^{43}$ or further migration of cells into the meshwork. A total of 3458 nuclei were measured. After 96 hours in culture, many seeded cells demonstrated large euchromatinic nuclei, indicating active transcription during infiltration.

\section{IOP homeostatic function of tissue-engineered ex vivo cultures}

After 24 hours of P scaffold perfusion, a stable IOP baseline was achieved (Fig. 5). No significant difference was found between decellularized (D) and reseeded (RS) groups at 
baseline $(D=11.81 \pm 0.52, R S=12.42 \pm 0.48 \mathrm{mmHg}, \mathrm{p}=0.40)$ or after cell seeding/sham $(D=$ $9.23 \pm 0.36, \mathrm{RS}=8.48 \pm 0.22 \mathrm{mmHg}, \mathrm{p}=0.07$ ). Conversely, IOP was highly unstable in scaffolds seeded with CrFK cells ( $\mathrm{CrFK}=27.0 \pm 17.3, \mathrm{TM}=8.48 \pm 2.67 \mathrm{mmHg}, \pm$ standard deviation.) At 48 hours post TM reseeding/sham, infusion rate was doubled from 3 to $6 \mu \mathrm{L} / \mathrm{min}$ to challenge the TM's IOP homeostatic response. Reseeded scaffolds maintained IOP within a healthy range, lower than in hypertensive decellularized scaffolds $(D=35.23 \pm 2.20, R S=16.53 \pm 0.89 \mathrm{mmHg}$, $\mathrm{p}<0.0001, \mathrm{~N}: 8,8$, Fig. 5.).

\section{Discussion}

In this study, we detail a tissue-engineered anterior segment organ culture model with functional similarities to ex vivo and in vivo outflow physiology. After an infusion rate increase challenge, anterior segments with recellularized TM maintained pressures of $16.5 \pm 0.9 \mathrm{mmHg}$ while decellularized meshwork had significantly higher pressures of $35.2 \pm 2.2 \mathrm{mmHg}$. Our results were on a timescale and of a magnitude similar to those observed in human anterior segment perfusion models with infusion rate doubling ${ }^{11}$ and after IOP increase ${ }^{44}$.

TM cellularity along existing trabecular beams decreases gradually with age ${ }^{45,46}$. Both increased age and decreased TM cellularity are major risk factors for the development of glaucoma. However, removal of meshwork tissue (both cells and ECM) is well-known to reduce IOP both ex vivo ${ }^{27}$ and clinically ${ }^{47-49}$. Here, we show that the presence of decellularized TM ECM is associated with significant, sustained IOP elevation after a homeostatic challenge. These findings are consistent with results that detail ECM remodeling by TM cells as a means of IOP regulation ${ }^{9-12}$.

Previously described glaucoma models in which about $1 / 3$ rd of meshwork cells were killed with saponin treatment demonstrated functional restoration after transplantation of human TM cells or TM-like iPSCs ${ }^{23}$. In the scaffolds presented here, $100 \%$ of cells were removed before cell transplantation. This allowed complete control over which cells end up in the scaffold. Functional responses are known to be caused by transplanted cells alone, and not the effects of transplanted cells on native ones. Additionally, because cell debris and detergents can be washed from the scaffold prior to cell-seeding, viable cells do not need to be subjected to 
potentially deleterious quantities of chemical stressors like saponin or lytic cell contents which may affect healthy TM cell physiology.

After 96 hours in culture, meshwork infiltration of seeded cells rendered comparable cell infiltration depth to controls. Due to the segmental nature of outflow and multilayer structure of the TM, treatments introduced intracamerally may not reach all meshwork cells evenly. For example, experimental manipulations, including lentiviral transduction of ex vivo TM is highly efficient ${ }^{24}$ but does not transduce all native cells. Reseeding cells cultured, treated, or sorted in vitro before transplantation allows for more thorough and exact modifications of cells and tissue.

Preliminary studies in which 1 million cells were seeded into scaffolds (Supplementary material 2), a cell number closer to that of a young, healthy $\mathrm{TM}^{46}$, resulted in low TE TM cellularity. This is consistent with cell loss percentages in other TE organs ${ }^{29,50}$. It is possible that seeded TM cells require sufficient cell-cell contact early in the process of meshwork infiltration and otherwise cannot survive or proliferate. In addition to the optimization of cell-loss, future studies can be done to test longer durations of culture as well as scaffold biocompatibility with human TM cells.

In conclusion, we describe the construction of a fully decellularized porcine anterior segment scaffold and successful reseeding with non-native meshwork cells. TE ex vivo cultures demonstrated localization of transplanted cells to the TM region, cell infiltration of ECM, and IOP homeostatic ability after infusion rate challenge. Tissue-engineered organ culture models may allow researchers to study TM cells in the context of a standard 3D environment with reduced need for human donor eyes.

\section{Methods}

A total of 41 anterior segments were prepared as previously reported ${ }^{20,51,52}$. Briefly, porcine eyes were acquired within 4 hours of sacrifice, decontaminated with povidone-iodine, and hemisected along the equator in an aseptic biosafety cabinet. The posterior segment, lens, and iris were carefully removed. 


\section{Scaffold production}

As described previously ${ }^{42,53}$, Freeze/thaw (F/th) anterior segments were sealed in an airtight container and cycled between $-80^{\circ} \mathrm{C}$ and room temperature two times to lyse all native cells $^{42,53}$. F/th scaffolds were then perfused with perfusion culture media (DMEM supplemented with $1 \%$ FBS and $1 X$ antibiotic/antimycotic) for 5 days $(n=4)$.

A time-course assay was conducted to determine the minimum necessary time to produce decellularized scaffolds via immersion/agitation-mediated decellularization (IA). F/th anterior segments were placed in SDS solution (0.01\% wt/vol in PBS + anti-anti) for 1 day $(n=3)$, 2 days $(n=3)$, and 5 days $(n=3)$. Following SDS incubations, solutions were changed to $0.1 \%$ TritonX-100 for 24 hours and then a perfusion culture media wash for 48 hours. Samples were affixed to a vertical stage rotated at 20 RPM during each incubation. Fluid exchanges were performed every 24 hours.

For perfusion-mediated decellularization $(\mathrm{P})$, we modified an existing matrix production protocol used in bioartificial heart construction ${ }^{29}$. F/th segments were maintained via constantrate perfusion ${ }^{21}$ at $6 \mu \mathrm{L} / \mathrm{min}$ with SDS solution for 24 hours, TritonX-100 solution for 24 hours, and perfusion culture media for 48 hours $(n=24)$. Untreated control samples were maintained for 5 days with perfusion culture media $(n=4)$.

\section{Cell culture}

Porcine TM culture was performed as done previously ${ }^{20,54}$. TM was dissected away from anterior segments under an ophthalmic operating microscope (Stativ S4, Carl Zeiss, Oberkochen, Germany) and cut into $0.5 \mathrm{~mm}^{3}$ segments. Tissue pieces were cultured in T25 flasks containing OptiMEM (31985-070, Gibco, Life Technologies, Grand Island, NY, USA), supplemented with 5\% FBS and antibiotic/antimycotic (15240062, Thermo Fisher Scientific, Waltham, MA, USA). Cells were passaged at $80 \%$ confluence and used for experiments at passages 2-4.

Due to limited vector stocks and unlimited passaging of cell-lines, a CrFK cell-line transduced with an eGFP GINSIN vector ${ }^{24,55}$ was used to determine initial scaffold 
biocompatibility and real-time tracking of seeded cell localization. GINSIN CrFK cells were maintained in $2.5 \%$ FBS DMEM + antibiotic/antimycotic and passaged at $80 \%$ confluence.

\section{Live-cell tracking}

Three million GINSIN CrFK cells were seeded into P scaffolds to determine biocompatibility and cell localization in real-time. A 20G cannula (BD PrecisionGlide 305176) attached to a $1 \mathrm{~mL}$ syringe (Norm-Ject Tuberkulin Luer 4010-200V0) was connected to a $3 \mathrm{~cm}$ length of perfusion tubing. The inlet and outlet of each perfusion dish were disconnected from the perfusion system and reconnected to the $1 \mathrm{~mL}$ syringes. A $200 \mathrm{uL}$ bolus of culture media was removed from anterior chambers through the outlet. A $200 \mathrm{uL}$ bolus of 3 million GINSIN-CrFK cells was slowly introduced into the anterior chamber. Cultures were gravity perfused at $15 \mathrm{mmHg}$ for 20 minutes. Dishes were positioned with corneas facing downward and perfusion culture was reinitiated.

TM was visualized from the underside of transparent perfusion dishes with an epifluorescence-equipped dissecting microscope (Olympus SZX16 with GFP filter cube and DP80 monochrome/color camera; Olympus Corp., Center Valley, PA, USA) and imaged at 24, 48, and 144 hours post-seeding. Mean fluorescent intensity of images was measured in four concentric regions (central cornea (1), peripheral cornea (2), TM (3), and sclera/ciliary body remnant (4), Fig. 3, bottom right) in Fiji ${ }^{56}$ and compared. A uniform threshold was applied to fluorescence images to aid visualization in Fig. 3, right panels.

\section{IOP maintenance challenge}

To test meshwork function restoration, decellularized scaffolds were perfused for 24 hours with TM culture media before seeding to achieve stable baseline IOPs. Three million porcine TM cells were seeded as described above in RS while D received a sham bolus of cellfree TM culture media. After 48 hours, the infusion rate was doubled from $3 \mu \mathrm{L} / \mathrm{min}$ to $6 \mu \mathrm{L} / \mathrm{min}$ to challenge the TM's IOP homeostatic response. 


\section{Histology}

Samples were fixed in 4\% PFA for 48 hours, embedded in paraffin, and sectioned at $6 \mu \mathrm{m}$ thickness. Sections were stained with H\&E for morphological analysis and DAPI for DNA content.

\section{Statistical analysis}

Fluorescence intensities of DAPI-labeled scaffold samples were compared to control. Live-cell eGFP fluorescence of the TM region was compared to all other locations. Cell depth at each time-point was compared to control. IOP recordings were sampled into 2-hour blocks, and D was compared to RS at each experimental phase (baseline, cell seeding, and challenge). Periods of 8 hours after media refilling were excluded from the analysis to allow for IOP stabilization. Statistical comparisons were conducted with a student's t-test in Python 3.6. Data are expressed as mean \pm SEM unless otherwise noted. P-values $<0.05$ were considered statistically significant.

\section{Acknowledgments}

This study was funded by the Initiative to Cure Glaucoma of the Eye and Ear Foundation of Pittsburgh (NAL), by NEI Grant K08EY022737, by NIH CORE Grant P30 EY08098 to the Department of Ophthalmology, and an unrestricted grant from Research to Prevent Blindness, New York, NY.

\section{Compliance with Ethical Standards}

This article does not contain any studies with human participants. Porcine eyes were acquired from a local abattoir; no animals were sacrificed for the purpose of this study. Approval by an ethics committee or institutional animal care and use committee was not required.

\section{Competing Interests}

The authors declare no competing interests. 


\section{Data Availability}

The datasets generated and analyzed during the current study are available from the corresponding author on reasonable request.

\section{Corresponding Author}

Correspondence to Nils A. Loewen 


\section{References}

1. Tham, Y.-C. et al. Global prevalence of glaucoma and projections of glaucoma burden through 2040: a systematic review and meta-analysis. Ophthalmology 121, 2081-2090 (2014).

2. Maus, T. L. \& Brubaker, R. F. Measurement of aqueous humor flow by fluorophotometry in the presence of a dilated pupil. Invest. Ophthalmol. Vis. Sci. 40, 542-546 (1999).

3. Goel, M., Picciani, R. G., Lee, R. K. \& Bhattacharya, S. K. Aqueous humor dynamics: a review. Open Ophthalmol. J. 4, 52-59 (2010).

4. Chan, M. P. Y. et al. Glaucoma and intraocular pressure in EPIC-Norfolk Eye Study: cross sectional study. BMJ 358, j3889 (2017).

5. Sommer, A. et al. Relationship between intraocular pressure and primary open angle glaucoma among white and black Americans. The Baltimore Eye Survey. Arch. Ophthalmol. 109, 1090-1095 (1991).

6. Nickells, R. W. et al. Surgical lowering of elevated intraocular pressure in monkeys prevents progression of glaucomatous disease. Exp. Eye Res. 84, 729-736 (2007).

7. Gordon, M. O. \& Kass, M. A. The Ocular Hypertension Treatment Study: design and baseline description of the participants. Arch. Ophthalmol. 117, 573-583 (1999).

8. Leske, M. C. et al. Predictors of long-term progression in the early manifest glaucoma trial. Ophthalmology 114, 1965-1972 (2007).

9. Bradley, J. M. et al. Effects of mechanical stretching on trabecular matrix metalloproteinases. Invest. Ophthalmol. Vis. Sci. 42, 1505-1513 (2001).

10. Fleenor, D. L. et al. TGFbeta2-induced changes in human trabecular meshwork: implications for intraocular pressure. Invest. Ophthalmol. Vis. Sci. 47, 226-234 (2006).

11. Acott, T. S. et al. Intraocular pressure homeostasis: maintaining balance in a high-pressure environment. J. Ocul. Pharmacol. Ther. 30, 94-101 (2014). 
12. Vranka, J. A. et al. Biomechanical Rigidity and Quantitative Proteomics Analysis of Segmental Regions of the Trabecular Meshwork at Physiologic and Elevated Pressures. Invest. Ophthalmol. Vis. Sci. 59, 246-259 (2018).

13. Zenkel, M. et al. Differential gene expression in pseudoexfoliation syndrome. Invest. Ophthalmol. Vis. Sci. 46, 3742-3752 (2005).

14. Torrejon, K. Y. et al. Bioengineered glaucomatous 3D human trabecular meshwork as an in vitro disease model. Biotechnol. Bioeng. 113, 1357-1368 (2016).

15. Torrejon, K. Y. et al. Recreating a human trabecular meshwork outflow system on microfabricated porous structures. Biotechnol. Bioeng. 110, 3205-3218 (2013).

16. Duval, K. et al. Modeling Physiological Events in 2D vs. 3D Cell Culture. Physiology 32, 266-277 (2017).

17. Kapałczyńska, M. et al. 2D and 3D cell cultures - a comparison of different types of cancer cell cultures. Arch. Med. Sci. 14, 910-919 (2018).

18. Huh, D., Hamilton, G. A. \& Ingber, D. E. From 3D cell culture to organs-on-chips. Trends Cell Biol. 21, 745-754 (2011).

19. Alford, P. W., Feinberg, A. W., Sheehy, S. P. \& Parker, K. K. Biohybrid thin films for measuring contractility in engineered cardiovascular muscle. Biomaterials 31, 3613-3621 (2010).

20. Dang, Y. et al. A porcine ex vivo model of pigmentary glaucoma. Sci. Rep. 8, 5468 (2018).

21. Loewen, R. T. et al. A Porcine Anterior Segment Perfusion and Transduction Model With Direct Visualization of the Trabecular Meshwork. Invest. Ophthalmol. Vis. Sci. 57, 1338-1344 (2016).

22. Sherwood, J. M., Reina-Torres, E., Bertrand, J. A., Rowe, B. \& Overby, D. R. Measurement of Outflow Facility Using iPerfusion. PLoS One 11, e0150694 (2016).

23. Abu-Hassan, D. W., Li, X., Ryan, E. I., Acott, T. S. \& Kelley, M. J. Induced pluripotent stem cells restore function in a human cell loss model of open-angle glaucoma. Stem Cells 33, 751-761 (2015). 
24. Loewen, N. et al. Genetic modification of human trabecular meshwork with lentiviral vectors. Hum. Gene Ther. 12, 2109-2119 (2001).

25. Fautsch, M. P. et al. Perfusion of his-tagged eukaryotic myocilin increases outflow resistance in human anterior segments in the presence of aqueous humor. Invest. Ophthalmol. Vis. Sci. 47, 213221 (2006).

26. Loewen, N. et al. Preservation of aqueous outflow facility after second-generation FIV vectormediated expression of marker genes in anterior segments of human eyes. Invest. Ophthalmol. Vis. Sci. 43, 3686-3690 (2002).

27. Dang, Y. et al. Outflow enhancement by three different ab interno trabeculectomy procedures in a porcine anterior segment model. Graefes Arch. Clin. Exp. Ophthalmol. 256, 1305-1312 (2018).

28. Swaminathan, S. S. et al. Histologic Analysis of Trabecular Meshwork Obtained from Kahook Dual Blade Goniotomy. Am. J. Ophthalmol. (2018). doi:10.1016/j.ajo.2018.05.028

29. Ott, H. C. et al. Perfusion-decellularized matrix: using nature's platform to engineer a bioartificial heart. Nat. Med. 14, 213-221 (2008).

30. Guan, Y. et al. Porcine kidneys as a source of ECM scaffold for kidney regeneration. Mater. Sci. Eng. C Mater. Biol. Appl. 56, 451-456 (2015).

31. Ott, H. C. et al. Regeneration and orthotopic transplantation of a bioartificial lung. Nat. Med. 16, 927-933 (2010).

32. Song, J. J. et al. Regeneration and experimental orthotopic transplantation of a bioengineered kidney. Nat. Med. 19, 646-651 (2013).

33. Dahl, S. L. M., Koh, J., Prabhakar, V. \& Niklason, L. E. Decellularized native and engineered arterial scaffolds for transplantation. Cell Transplant. 12, 659-666 (2003).

34. Lin, P., Chan, W. C. W., Badylak, S. F. \& Bhatia, S. N. Assessing porcine liver-derived biomatrix for hepatic tissue engineering. Tissue Eng. 10, 1046-1053 (2004). 
35. Macchiarini, P. et al. Clinical transplantation of a tissue-engineered airway. Lancet 372, 2023-2030 (2008).

36. Wainwright, D. J. Use of an acellular allograft dermal matrix (AlloDerm) in the management of fullthickness burns. Burns 21, 243-248 (1995).

37. Bejjani, G. K., Zabramski, J. \& Durasis Study Group. Safety and efficacy of the porcine small intestinal submucosa dural substitute: results of a prospective multicenter study and literature review. J. Neurosurg. 106, 1028-1033 (2007).

38. Eichler, C. et al. A Head to Head Comparison Between SurgiMend ${ }^{\circledR}-$-Fetal Bovine Acellular Dermal Matrix and Tutomesh ${ }^{\circledR}$--A Bovine Pericardium Collagen Membrane in Breast Reconstruction in 45 Cases. In Vivo 31, 677-682 (2017).

39. Guyette, J. P. et al. Bioengineering Human Myocardium on Native Extracellular Matrix. Circ. Res. 118, 56-72 (2016).

40. Schaner, P. J. et al. Decellularized vein as a potential scaffold for vascular tissue engineering. J. Vasc. Surg. 40, 146-153 (2004).

41. Gerli, M. F. M., Guyette, J. P., Evangelista-Leite, D., Ghoshhajra, B. B. \& Ott, H. C. Perfusion decellularization of a human limb: A novel platform for composite tissue engineering and reconstructive surgery. PLoS One 13, e0191497 (2018).

42. Dang, Y. et al. Freeze-thaw decellularization of the trabecular meshwork in anex vivoeye perfusion model. PeerJ 5, e3629 (2017).

43. Polansky, J. R., Weinreb, R. N., Baxter, J. D. \& Alvarado, J. Human trabecular cells. I. Establishment in tissue culture and growth characteristics. Invest. Ophthalmol. Vis. Sci. 18, 1043-1049 (1979).

44. Borrás, T., Rowlette, L. L. S., Tamm, E. R., Gottanka, J. \& Epstein, D. L. Effects of elevated intraocular pressure on outflow facility and TIGR/MYOC expression in perfused human anterior segments. Invest. Ophthalmol. Vis. Sci. 43, 33-40 (2002). 
45. McMenamin, P. G., Lee, W. R. \& Aitken, D. A. Age-related changes in the human outflow apparatus. Ophthalmology 93, 194-209 (1986).

46. Alvarado, J., Murphy, C., Polansky, J. \& Juster, R. Age-related changes in trabecular meshwork cellularity. Invest. Ophthalmol. Vis. Sci. 21, 714-727 (1981).

47. Dang, Y.-L. et al. Two-year outcomes of ab interno trabeculectomy with the Trabectome for Chinese primary open angle glaucoma: a retrospective multicenter study. Int. J. Ophthalmol. 11, 945-950 (2018).

48. Berdahl, J. P. et al. Six-Month Outcomes of Goniotomy Performed with the Kahook Dual Blade as a Stand-Alone Glaucoma Procedure. Adv. Ther. 35, 2093-2102 (2018).

49. Esfandiari, H. et al. Five-year clinical outcomes of combined phacoemulsification and trabectome surgery at a single glaucoma center. Graefes Arch. Clin. Exp. Ophthalmol. (2018). doi:10.1007/s00417-018-4146-y

50. Wendt, D., Marsano, A., Jakob, M., Heberer, M. \& Martin, I. Oscillating perfusion of cell suspensions through three-dimensional scaffolds enhances cell seeding efficiency and uniformity. Biotechnol. Bioeng. 84, 205-214 (2003).

51. Waxman, S. et al. Structure-Function Changes of the Porcine Distal Outflow Tract in Response to Nitric Oxide. Invest. Ophthalmol. Vis. Sci. 59, 4886-4895 (2018).

52. Dang, Y. et al. RKI-1447, a Rho kinase inhibitor, causes ocular hypotension, actin stress fiber disruption, and increased phagocytosis. Graefes Arch. Clin. Exp. Ophthalmol. 257, 101-109 (2019).

53. Burk, J. et al. Freeze-thaw cycles enhance decellularization of large tendons. Tissue Eng. Part C Methods 20, 276-284 (2014).

54. Wang, C. et al. Impact of pigment dispersion on trabecular meshwork cells. Graefes Arch. Clin. Exp. Ophthalmol. (2019). doi:10.1007/s00417-019-04300-7

55. Zhang, Z. et al. Outflow tract ablation using a conditionally cytotoxic feline immunodeficiency viral 
vector. Invest. Ophthalmol. Vis. Sci. 55, 935-940 (2014).

56. Schindelin, J. et al. Fiji: an open-source platform for biological-image analysis. Nat. Methods 9, 676682 (2012).

\section{Author Contributions}

SW: manuscript writing with input from all co-authors, data collection, data analysis, figure creation, contributed to study design. CW: experimentation, data collection, reviewed manuscript. YD: experimentation, reviewed manuscript. RL: experimentation, reviewed manuscript. NAL: experimentation, funding, study design, and oversaw data collection, manuscript writing. 


\section{Figures}

\section{Figure 1: freeze/thaw + surfactant perfusion decellularized AS scaffolds}

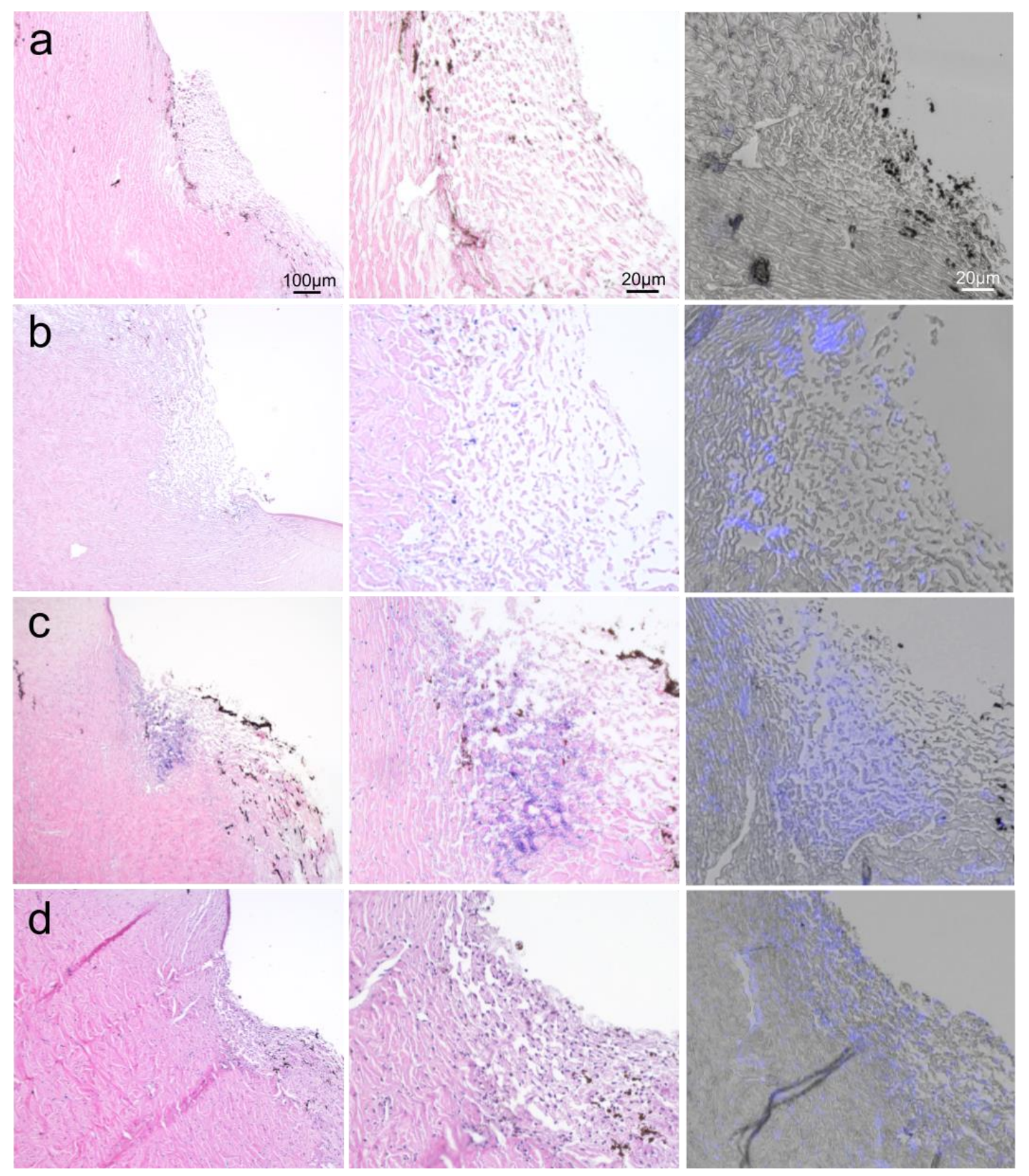

Fig. 1: Freeze/thaw cycling + surfactant perfusion (a), Freeze/thaw + surfactant agitation (b), Freeze/thaw (c), and untreated control (d). Left: H\&E staining, middle: magnified view of TM in left, right: DAPI + brightfield. 
Figure 2: IOP within physiological range throughout perfusion-decellularization

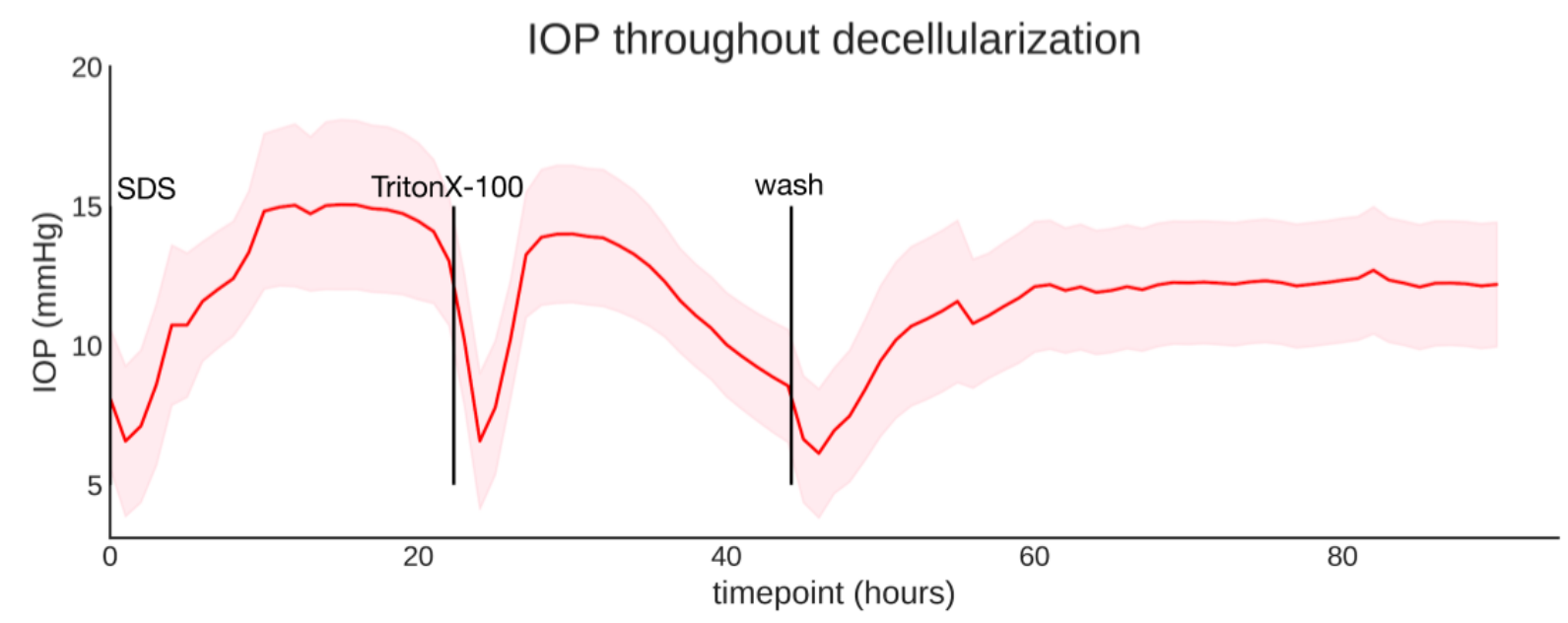

Fig 2: IOP remains within a physiological range throughout perfusion decellularization $(12.27 \pm 0.04 \mathrm{mmHg}, \mathrm{n}=15)$. Brief IOP drops after media exchanges are due to lapse in perfusion during a system restart. Error fields indicate SEM. 


\section{Figure 3: Seeded cells localized to the TM region}

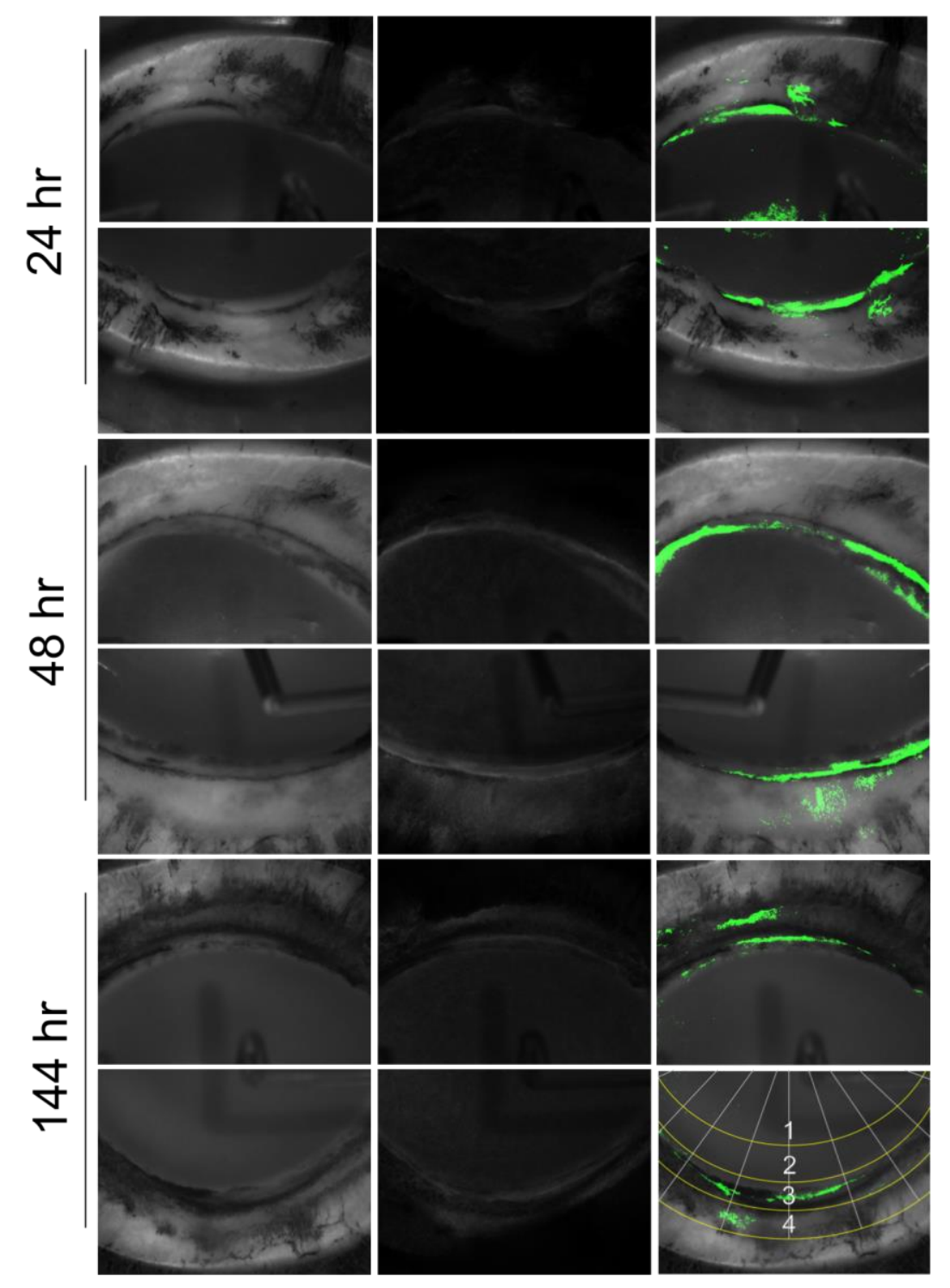

Fig. 3: Seeded cells localized to the TM region. Anterior segment scaffolds seeded with 3 million eGFP CrFK cells as visualized from the underside of clear perfusion culture dishes. Left:

brightfield view of TM angle, middle: eGFP fluorescence, right: merged. Localization of intensity in fluorescence images was evaluated in the 4 concentric regions shown at the bottom right panel (central cornea (1), peripheral cornea (2), TM (3), and sclera/ciliary body remnant (4).) TM was brighter than all other regions $(90.1 \pm 7.5 \%$ brighter, $\mathrm{p}<0.001$.) 
Figure 4: Cell infiltration of the TM increases with time in perfusion culture
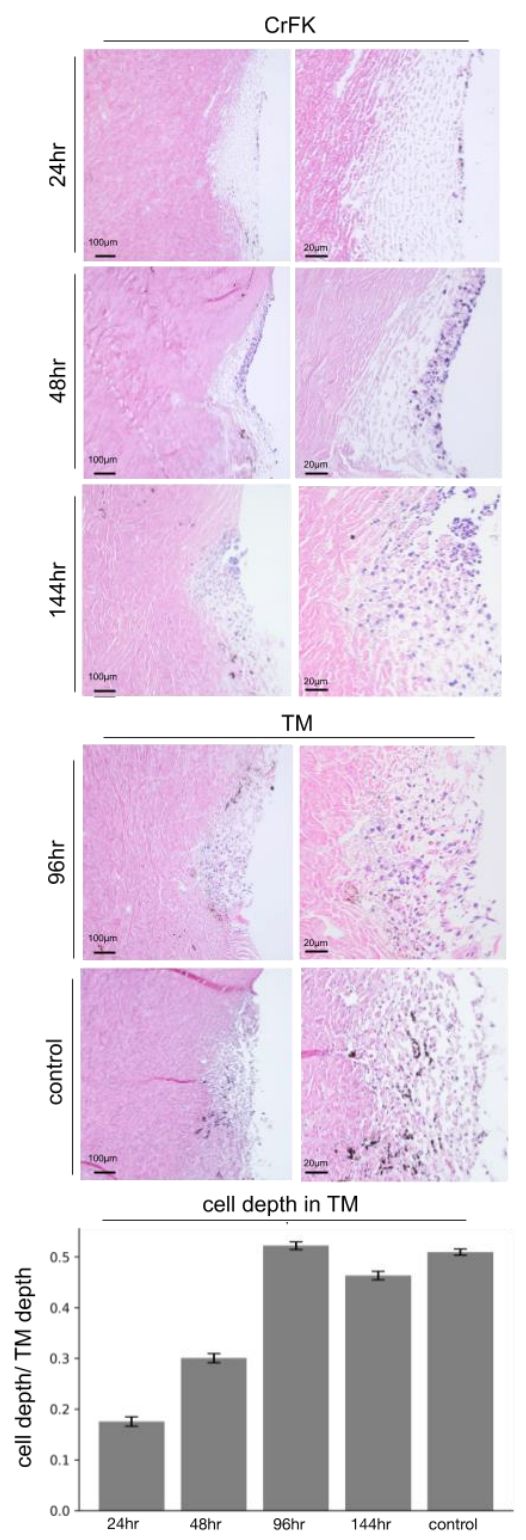

Fig. 4: Cell infiltration of the TM at 24, 48 , and 144 hours for cultures reseeded with CrFK cells shown in Fig. 3. Cell infiltration of scaffolds reseeded with porcine TM cells is shown at 96 hrs, after infusion rate increase challenge. Cell depth at 24 and 48 hours was significantly different from control $(p<0.001)$ while cell depth at 96 and 144 hours and above was not significantly different $(p=0.19,0.82)$. Error bars indicate SEM. 
Figure 5: Tissue-engineered ex vivo cultures demonstrate IOP homeostatic function
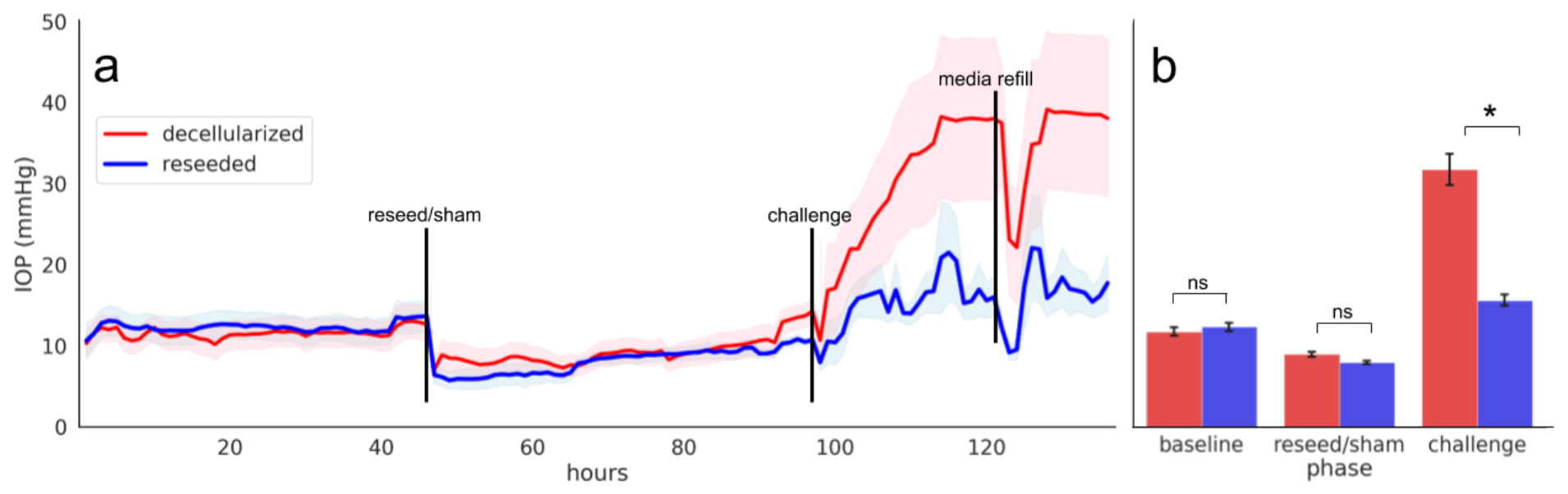

Fig. 5: Tissue-engineered ex vivo cultures demonstrate IOP homeostatic function. A) Time-series IOP data of decellularized and reseeded scaffolds. B) Summary of cleaned IOP at each experimental phase. No significant difference was found between decellularized (D) and reseeded $(R S)$ at baseline $(p=0.40)$ or after cell seeding/sham $(p=0.07)$. After 48 hours, the infusion rate was doubled from $3 \mu \mathrm{L} / \mathrm{min}$ to $6 \mu \mathrm{L} / \mathrm{min}$ to challenge the TM's IOP homeostatic response. RS maintained IOP within a healthy range while D was significantly elevated ( $p<$ $0.0001, \mathrm{~N}: 8,8$.) Error fields indicate SEM, ns= not significant, $* p>0.0001$. 


\section{Tables}

Table 1: Cell infiltration of scaffolds

\begin{tabular}{cccccc}
\hline timepoint & 24 hours & 48 hours & 96 hours & 144 hours & control \\
\hline cell type & CrFK & CrFK & TM & CrFK & TM \\
$\begin{array}{c}\text { depth in } \\
\text { meshwork (\%) }\end{array}$ & $17.5 \pm 0.9^{*}$ & $30.0 \pm 0.9^{*}$ & $52.2 \pm 0.7$ & $49.1 \pm 0.7$ & $50.9 \pm 0.6$ \\
cellularity & $9.2 \pm 1.6^{*}$ & $23.1 \pm 4.4^{*}$ & $60.1 \pm 6.4^{*}$ & $60.0 \pm 13.0$ & $100.0 \pm 11.1$ \\
(per section) & & & & &
\end{tabular}

Table 1: Cell infiltration of scaffolds. Depth of infiltrated cells is shown relative to the complete depth of the meshwork. TE TM cellularity values at each time-point are normalized to control. Error indicates SEM. Asterisk indicates significant difference from respective control $(p<0.05)$. 


\section{Supplemental material}

\section{Supplemental material 1: Map of materials and methods}

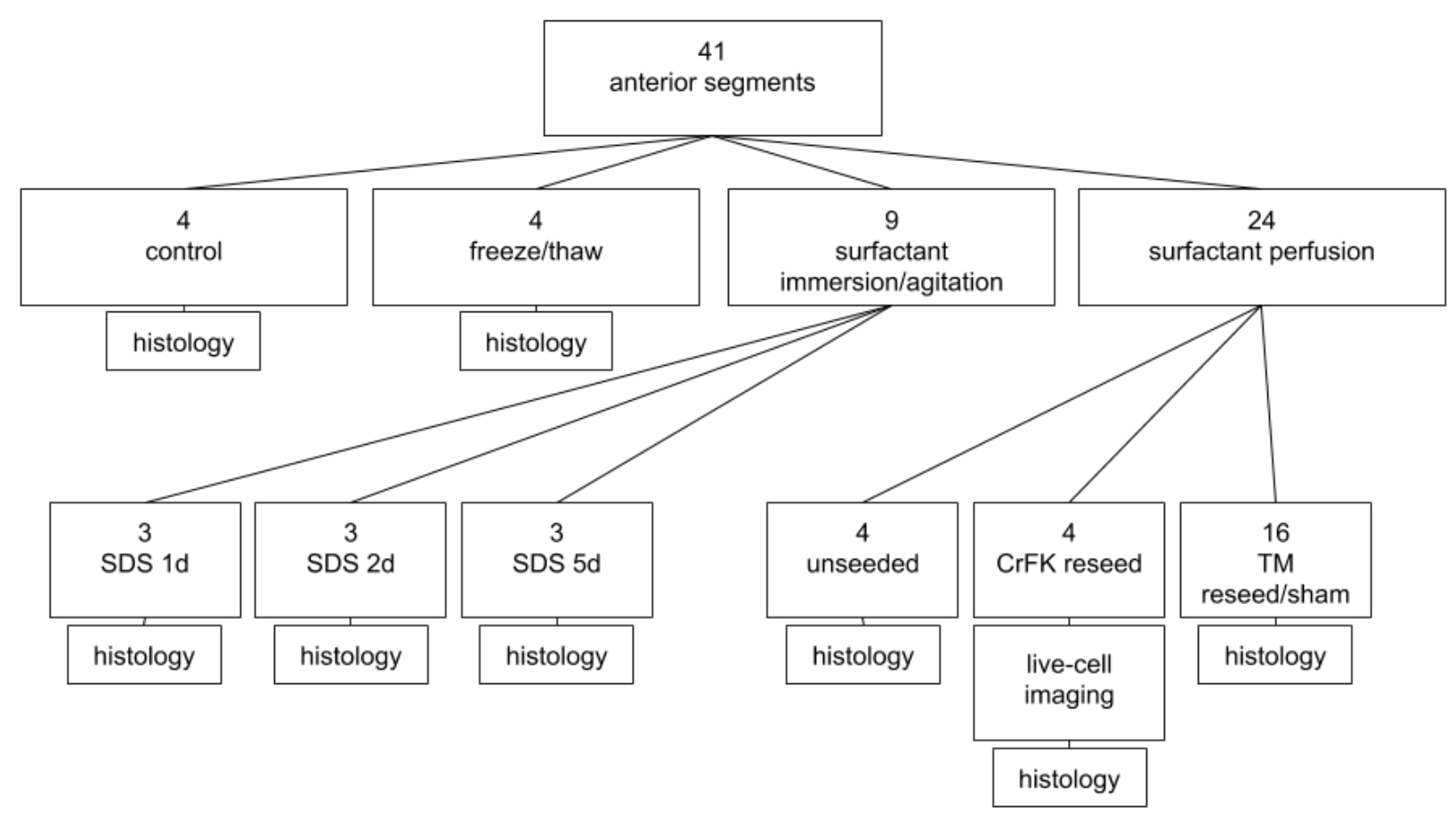

